\title{
Comparison of Systolic Period Duration Using Aortic Flow or Pressure Based Methods in Anesthetized Patients
}

\author{
Arthur Le Gall ${ }^{1,2,3}$, Alexandre Laurin ${ }^{1,2}$, Fabrice Vallee $^{1,2,3}$, Denis Chemla $^{4}$ \\ ${ }^{1}$ Inria, Université Paris-Saclay, Palaiseau, France \\ ${ }^{2}$ LMS, École Polytechnique, CNRS, Université Paris-Saclay, Palaiseau, France \\ ${ }^{3}$ Anesthesiology - Intensive care \& SMUR department, Saint-Louis \& Lariboisiere Hospitals, \\ Assistance - Publique / Hopitaux de Paris, Paris, France \\ ${ }^{4}$ Cardio-Vascular Physiology department, Kremlin-Bicetre Hospital, Assistance-Publique/Hopitaux \\ de Paris - INSERM UMR-S999, Paris, France
}

\begin{abstract}
It has been shown that Systolic Period Duration (SPD) measured with trans-esophageal echocardiography (TEE) is significantly shorter compared to measurements made with radial arterial pressure waveform. This difference could be interpreted in terms of arterial pressure amplification, a mechanism by which arterial pressure waveform is transformed alongside the arterial tree. This amplification is closely related to cardiac afterload as it shares common determinants, like arterial stiffness, pulse wave velocity, or aortic reflection waves.

In turn, afterload estimated partly using arterial pressure amplification is useful during general anesthesia (GA) to evaluate detrimental or beneficial effects of vasopressors given to combat hypotensions. Despite TEE measurements comport some impracticalities, trans-esophageal Doppler (TE Doppler) is routinely used for cardiac output monitoring.

The goal of this paper was to compare SPD measured with TE Doppler and with arterial pressure at the same location. A secondary goal was to describe a novel dicrotic notch identification algorithm that uses the interspace between the pressure waveform and the straight line going from the systolic peak of one beat to the foot of the subsequent beat.

Twenty three patients undergoing GA for neurointerventional procedures were included. Central pressure was obtained by fluid filled radiologic guidewire connected to a pressure transducer. Flow velocity waveform was obtained using TE Doppler probe inserted in the esophagus after induction of GA. Pressure and flow velocity measurements were recorded simultaneously when the tip of the pressure catheter was placed in front of the Doppler probe, in the descending Aorta.
\end{abstract}

SPD measured from Flow velocity waveform was signif- icantly shorter than from Pressure waveform $(343 \pm 46 \mathrm{~ms}$ vs $415 \pm 54 \mathrm{~ms} ; p<0.001$ ).

Flow velocity and Pressure waveform methods are not interchangeable. To measure SPD consistently at different locations, a consistent algorithm should be used, such as automated identification of dicrotic notch.

\section{Introduction}

Systolic period duration (SPD), computed from the foot and dicrotic notch times of arterial pressure waveforms, could be used to quantify arterial pressure amplification [1]. These shape variations that pressure waveforms undergo while travelling through the arterial tree have themselves been used as a surrogate of cardiac afterload (CA) [2]. Moreover, SPD has been shown to be significantly shorter in cases of increased CA [3].

During surgical procedures, general anesthesia leads to profound alterations of vascular tone, which is of interests to anesthesiologists who regularly administer vasopressors to combat anesthesia-induced hypotensions. While the magnitude and duration of hypotensions have been linked to post-operative complications [4], the beneficial effects of vasopressors are more tricky to assess. CA-mediated alteration of regional blood flow as a potential side-effect could lead to potential harms [5]. Accurate monitoring of CA thus emerges as an important practice when using vasopressors to prevent their detrimental effects. Novel methods of CA monitoring from routinely-acquired signals in the operating theatre have recently been proposed [6].

Arterial pressure amplification leads to increased systolic and decreased diastolic pressure. Mechanisms tuning the magnitude of arterial pressure amplification are linked to vascular pathophysiology, such as alterations of arterial stiffness, pulse wave velocity, reflected waves, and artery 

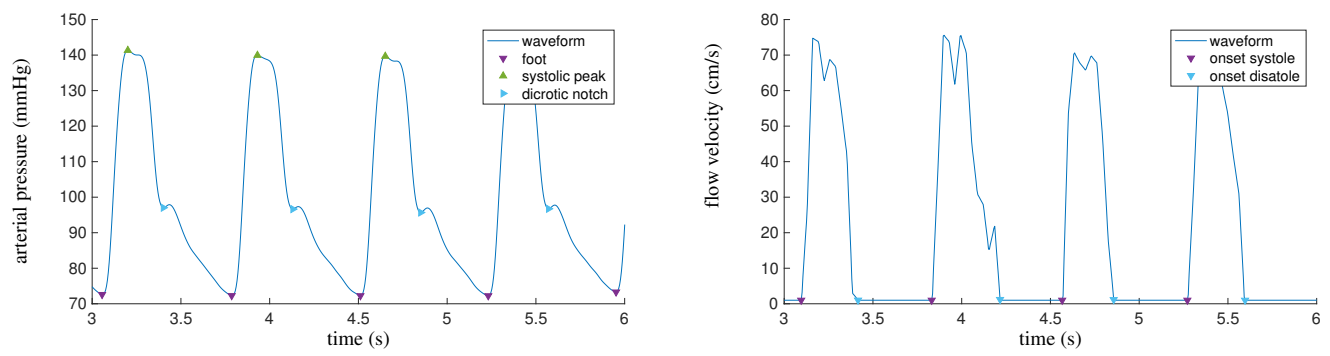

Figure 1. Example of annotated concurrent aortic pressure and flow velocity signals. Left: arterial pressure measured with a pressure transducer in the descending aorta; Right: flow velocity measured with trans-oesophageal Doppler probe in the descending aorta

elastic moduli, all of which are components of cardiac afterload [7].

Currently, peripherally measured SPD is defined as the duration from the foot of the waveform beat to the subsequent dicrotic notch. The reference method for the centrally measured ejection time involves transoesphageal echocardiography (TEE) [8], with continuous wave Doppler measurement through the Aortic valve. Although this technique is the most direct, it has the important limitation of not being available continuously. Transoesophageal Doppler (TE Doppler), a non invasive device inserted orally, allows continuous blood flow velocity measurement in the descending Aorta and could be an alternative to the TEE measurement of ejection period.

In a recent publication, Obata et al. [1] have observed a discrepancy between centrally and peripherally measured SPD in patients undergoing general anesthesia. Authors have hypothesized that this finding could be linked to the arterial pressure amplification mechanism.

To allow the isolation of the effect of arterial pressure amplification on the modification of centrally to peripherally measured SPD, we compared SPD obtained from TE Doppler with SPD measured simultaneously and at the same location by an arterial catheter.

\section{Material and Methods}

Patients undergoing neurosurgial procedure were included in the study. General anaesthesia was induced with Propofol $[3-6] \mathrm{mg} / \mathrm{l}$ and Remifentanil $[3-6]$ $\mathrm{ng} / \mathrm{l}$ in Targetted Controlled Infusion mode. Intubation was performed 3 minutes after $0.5 \mathrm{mg} / \mathrm{kg}$ body weight of Atracurium. Minute-Ventilation was set with Tidal Volume of $6-8 \mathrm{ml} / \mathrm{kg}$ and a Respiratory rate of 12 cyles per minute and adapted to reach a end Tidal Carbon Dioxide of $32-35 \mathrm{mmHg}$. After general anaesthesia induction, a TE Doppler probe (Deltex Medical, Chichester, UK) was inserted and the signal was focused. A low bypass filter was then applied in order to allow automated detection of systolic and diastolic periods.

During the interventional puncture, the neuro-radiologist inserted a radio-opaque guidewire inside the arterial tree, through which radiographic dye, and fluid could be administered. The procedure involved the displacement of the guidewire from the femoral to the intracranial arteries, passing through the iliac artery, abdominal aorta, thoracic descending aorta, thoracic ascending aorta, common carotid artery and internal carotid artery. This guidewire was fluid filled with saline isochloride in order to prevent gas embolus to flow into circulation. A pressure transducer was then connected to the guidewire in order to record arterial pressure signal when the catheter tip was placed into the thoracic descending aorta (Central Arterial Pressure), in front of the TE Doppler probe.

Cardiovascular monitoring devices were connected, through Philips MP 60 monitor, to a software - Ixtrend, Ixcellence - , allowing us to record continuously and simultaneously dedicated signals (Central Arterial Pressure and Aortic Flow Waveforms).

Arterial pressure indices were obtained using opensource annotation software [9]. The foot of each central pressure beat was defined by initial identification the maximum of the second derivative and subsequent identification of the local minimum of the waveform (see fig. 1). Each systolic peak was then identified as the point of maximal pressure between each sequential pair of feet. The dicrotic notch was defined as the point of highest vertical distance between the waveform itself and the straight line going from one systolic peak to the foot of the next beat (Figure 2). For $t_{i}^{s}$ and $t_{i+1}^{f}$ the time of the systolic peak and foot of 2 successive beats, the time of the dicrotic notch $t_{i}^{d}$ was defined as the time $t$ that maximized the function

$$
d(t)=\max \left(S \cdot t+\frac{P\left(t_{i}^{s}\right)}{S \cdot t_{i}^{s}}-P(t)\right),
$$

where $S$ was the slope $\frac{P\left(t_{i}^{s}\right)-P\left(t_{i+1}^{f}\right)}{t_{i}^{s}-t_{i+1}^{f}}, P$ was pressure, and 
$t \in\left[t_{i}^{s}, t_{i+1}^{f}\right]$.

The onset of systole and diastole on flow velocity time series were then defined as the first point where flow was greater or lower than $5 \mathrm{~cm} / \mathrm{s}$ in a $100 \mathrm{~ms}$ radius window around each foot and dicrotic notch, respectively.

Heart period was defined as the time between successive feet on arterial pressure waveforms and successive systole onset points on flow velocity waveforms. Systolic period was defined on arterial pressure waveform as the time from foot to notch, and on flow from systole onset to diastole onset.

Signal analysis was performed on Matlab 2016b (Mathworks inc, Massachusetts, USA), statistical tests were performed on JMP 12 (SAS Institute, North Carolina, USA). Data are expressed as mean \pm standard deviation unless otherwise indicated.

Ethical commitee of the French Society of Intensive Care Medicine have approved this protocol (CE-SRLF $14-34)$

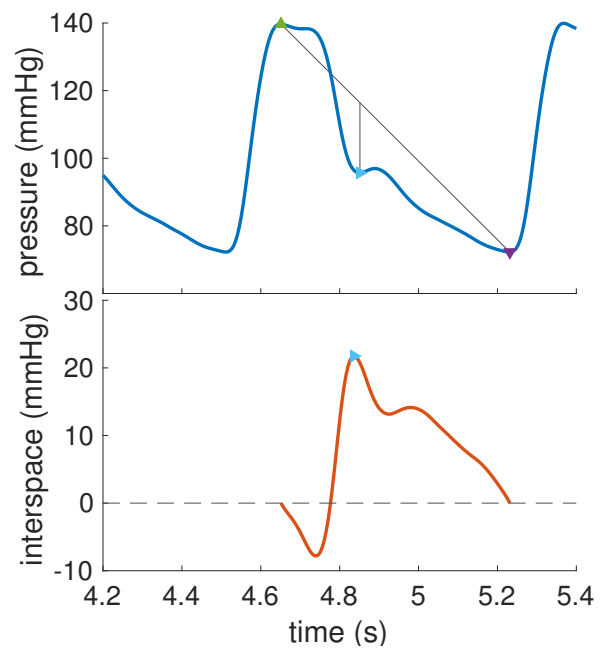

Figure 2. The dicrotic notch was automatically identified on the arterial pressure waveform as the point of highest vertical distance between the waveform itself and the straight line going from one systolic peak to the foot of the next beat. When a local minimum existed, it always occurred at this location.

\section{Results}

Twenty three patients were included in the study. Population characteristics are presented in table 1. Exclusion of intracranial aneurysm was the most frequent reason for neurointerventionnal procedure $(\mathrm{n}=13(56 \%))$, followed by delayed cerebral ischemia after sub-arachnoid haemmorrhage $(\mathrm{n}=3(13 \%))$, dural arteriovenous fistula or arterio-venous malformation $(\mathrm{n}=4(17 \%))$. Patients were predominantly young women without major risk of death as assessed by ASA classification.

Heart period obtained from the two methods were not significantly different $(945 \pm 203 \mathrm{~ms}$ vs $946 \pm 202 \mathrm{~ms}$; NS). SPD was significantly shorter when obtained from flow velocity compared to arterial pressure $(343 \pm 46 \mathrm{~ms}$ vs $415 \pm 54 \mathrm{~ms} ; p<0.001)$. Moreover, within individuals, SPD was consistently shorter when obtained from flow velocity compared to arterial pressure for 20 patients (91\%). Figure 3 is the SPD Bland-Altman plot. We observed a mean difference of $72 \mathrm{~ms}\left(\mathrm{CI}_{95 \%}=[49-95] \mathrm{ms}\right)$.

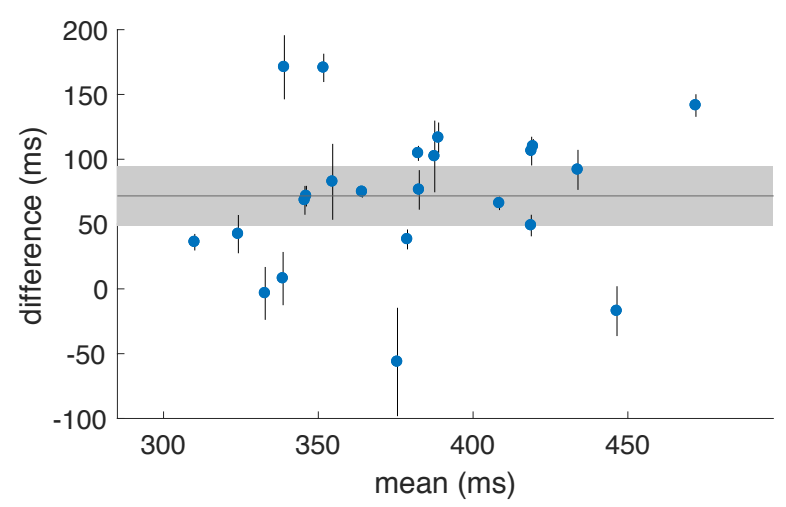

Figure 3. Bland-Altman plot comparing systolic time measured by the pressure and flow methods. Individual values are represented by the blue points superimposed on black vertical lines representing individual confidence intervals (CI). The population CI is represented by the horizontal grey band and the mean by a horizontal black line.

\section{Discussion}

Aortic pressure and flow velocity signals obtained during general anesthesia for neurointerventionnal procedure were analysed. Systolic period was computed for each heart beat: 1. as the time between the foot and notch of pressure waveforms and 2. as the time between the onset of systole and onset of diastole on flow velocity waveforms.

The main finding of this study is that despite the two methods allowed for accurate identification of cardiac period, SPD measurement by one or the other of the two methods were not interchangeable. Indeed, significant differences were found for the population means as well as in 20/23 participants. A consistent method of SPD identification is particularly important to estimate cardiac afterload by the mean of central to peripheral discrepancy of systolic period. This finding highlights Obata et al. [1] who found differences between central systolic period measured by TEE Doppler and peripheral systolic period measured by intra-artery transducer method. Authors postulated a possible interaction of the amplification process to explain this 
Table 1. Description of the population.

\begin{tabular}{lrc}
\hline \hline & & $\begin{array}{c}\text { Population } \\
n=23\end{array}$ \\
\hline Demographic Data & & \\
\hline Age & $(\mathrm{yo})$ & $49 \pm 14$ \\
Sex male & $\mathrm{n}(\%)$ & $11(48)$ \\
Weight & $(\mathrm{kg})$ & $73 \pm 14$ \\
Height & $(\mathrm{cm})$ & $169 \pm 10$ \\
ASA & & \\
$\quad$ I & $\mathrm{n}(\%)$ & $3(17)$ \\
II & $\mathrm{n}(\%)$ & $10(56)$ \\
III & $\mathrm{n}(\%)$ & $3(17)$ \\
IV & $\mathrm{n}(\%)$ & $0(0)$ \\
U & $\mathrm{n}(\%)$ & $5(28)$
\end{tabular}

\begin{tabular}{lrc} 
Hemodynamic Data & & \\
\hline Mean Pressure & $(\mathrm{mmHg})$ & $85 \pm 10$ \\
Heart Rate & $(\mathrm{BPM})$ & $63 \pm 11$ \\
Stroke Volume & $(\mathrm{ml})$ & $111 \pm 35$ \\
Systolic Pressure & $(\mathrm{mmHg})$ & $119 \pm 15$ \\
Diastolic Pressure & $(\mathrm{mmHg})$ & $66 \pm 9$
\end{tabular}

ASA: American Society of Anaesthesiology Classification; U: emergent surgery

difference. Methodological limitations of TEE Doppler acquisition and signal quality were also discussed. Our results support the hypothesis that technical problems between the two methods are prominent rather than various physiological processes. Indeed, the differences measured in [1] were present in our study even if with flow velocity and pressure measured at the same location.

Although it might constitute an abuse of language to call systolic period the time interval from foot to notch on a peripheral pressure waveform, its measurement is most certainly useful [7]. For the comparison of central vs peripheral systolic period, It might be preferable to use the same method in both locations.

The second objective of our study was to describe a novel dicrotic notch identification algorithm. The dicrotic notch was defined as the point of highest vertical interspace between the waveform itself and the straight line going from one systolic peak to the foot of the next beat [9]. Although no study was performed to test the accuracy of this automatic identification with respects to hand annotations performed by a team of experts, it corresponds to the accepted local-minimum definition when it applies. Furthermore, an automatic annotation has the benefit of being rigorously consistent.

Our study suffers from several limitations. We used TE Doppler to measure flow velocity. This methods comports several well-known presents technical issues. The post processing firmware in the device, designed to improve signal/noise ratio, involves filtering that can prematurely end the flow signal in late systole and otherwise modify exact timing measurements. Compared to the TEE method, TE Doppler is less operator dependant, but cannot be used to monitor velocities directly at the root of the aorta.

We didn't analyse the central to peripheral amplification as measured by the two methods. This analysis is currently being performed.

\section{Conclusion}

Measuring systolic period is used to quantify arterial pressure amplification, itself a surrogate of cardiac afterload. As measurements methods are subject to noise and technical difficulties, comparisons of method to identify onset and end of the systole are required. Our results suggests that systolic period measured with flow velocity and arterial pressure methods are not interchangeable, even when the measurements are made at the same location.

\section{References}

[1] Obata Y, Barodka V. Ejection time: influence of hemodynamics and site of measurement in the arterial tree, March 2017.

[2] Nichols W. Clinical measurement of arterial stiffness obtained from noninvasive pressure waveforms, January 2005.

[3] Salvi P, Benetos A. Left ventricular ejection time, not heart rate, is an independent correlate of aortic pulse wave velocity, December 2013.

[4] Bijker JB, Kalkman CJ. Intraoperative Hypotension and 1Year Mortality after Noncardiac Surgery:, December 2009.

[5] Thiele RH, Lynch C. The Clinical Implications of Isolated Alpha1 Adrenergic Stimulation:, August 2011.

[6] Valle F, Gayat E. Beat-by-beat assessment of cardiac afterload using descending aortic velocitypressure loop during general anesthesia: a pilot study, January 2017.

[7] Chirinos JA, Segers P. Noninvasive Evaluation of Left Ventricular Afterload: Part 2: Arterial Pressure-Flow and Pressure-Volume Relations in Humans, October 2010.

[8] Swaminathan M, Mathew JP. An Assessment of Two Different Methods of Left Ventricular Ejection Time Measurement by Transesophageal Echocardiography:, September 2003.

[9] Laurin A, Joachim J. Implementation of a feature detection algorithm for arterial blood pressure., 2017. URL https://fr.mathworks.com/matlabcentral/ fileexchange/60172-bp-annotate.

Address for correspondence:

Dr. Arthur Le Gall

Inria Paris-Saclay, Paris-Saclay University

Turing Building

2 rue Honore d'Estienne d'Orves, 91120, Palaiseau France arthur.le-gall@inria.fr 